\title{
Melanogenesis Inhibition by Mono-hydroxycinnamic Ester Derivatives in B16 Melanoma Cells
}

\author{
Kyung Dong Kim, Min Ho Song, Eul Kgun Yum, ${ }^{*}$ Ok Seon Jeon, ${ }^{\dagger}$ Young Woon Ju, ${ }^{\dagger}$ and Moon Sik Chang ${ }^{\dagger}$ \\ Department of Chemistry, Chungnam National University, Daejon 305-764, Korea. *E-mail: ekyum@cnu.ac.kr \\ ${ }^{\dagger} R \& D$ Center, Naturalsolution Corporation, Suwon 443-813, Korea \\ Received September 21, 2009, Accepted November 6, 2009
}

Key Words: Hydorxycinnamic esters, Benzylic, Depigmenting, Melanogenesis, Cytotoxicity

\begin{abstract}
Melanin is a human skin pigment produced in the melanosome, a unique intracytoplasmic organelle of melanocytes. ${ }^{1}$ The level of melanin synthesis determines the degree of skin pigmentation. ${ }^{2}$ Thus, controlling melanin synthesis is an important strategy in treating abnormal skin pigmentations, such as melanoma, freckles, and dark spots. ${ }^{3}$ In melanogenesis, melanin is the final product passing through numerous biological pathways and complex processes; at least 125 distinct genes are involved in the regulation of melanogenesis either directly or indirectly. ${ }^{4-6}$ Therefore, Measurement of the melanin content in melanocytes has been used as general means to examine depigmenting agents. ${ }^{7-9}$ Although numerous synthetic natural compounds, such as hydroquinone and retinoic acid, have been reported as skin depigmenting agents, ${ }^{10}$ serious side effects and cytotoxicity have limited their cosmetic application. The 'return to nature' trend in recent years has been accompanied by a booming interest in depigmenting agents from natural products. ${ }^{11}$ Representative synthetic natural product analogues that are currently in use in the depigmenting industry include arbutin and kojic acid derivatives. ${ }^{12,13}$

Many research groups have undertaken the synthesis of natural product analogues to find effective melanogenesis inhibitors for use as cosmetic ingredients. Our previous results with a 2,4-dihydroxycinnamic ester prompted us to examine the inhibitory activity of mono-hydroxycinnamic esters on melanogenesis and evaluate their potential as new effective depigmenting agents. ${ }^{14}$ Natural hydroxycinnamic acid can be obtained from various plants, such as Panax ginseng and Cinnamomum cassia Blume, and has been studied by food and drug researchers for its monophenolase and diphenolase activities. ${ }^{15-17}$ Specifically, considerable interest has been expressed in $p$-hydroxycinnamic acid due to its structural similarity with tyrosine. Despite the interesting results of hydroxycinnamic acid derivatives in food and drug research, investigation of their use as depigmenting agents in the cosmetic industry has been limited.

In our continuing research on hydroxycinnamic acid, we have synthesized benzylic mono-hydroxycinnamic substituted esters and tested their inhibitory effects on melanin synthesis within a non-cytotoxic concentration range using B16 melanoma cells.
\end{abstract}

\section{Results and Discussion}

The general synthetic pathway for mono-hydroxycinnamic ester derivatives is outlined in Figure 1. Hydroxycinnamic acid reacted with a phenyl-substituted alkyl bromide in the presence of $\mathrm{KHCO}_{3}$ at room temperature. The reaction conditions provided reasonable yields of several substituted benzyl bromides. The phenyl substituents were chosen considering electronic effects. ${ }^{18,19}$ Mono-hydroxycinnamic acids, kojic acid, and arbutin were tested as reference compounds for activity comparisons.

The cytotoxicity of the compounds was determined by MTT assay. The degree of inhibitory activity on melanin synthesis was determined using the melanin content assay at the low cytotoxicity concentration $(100 \mu \mathrm{M})$. The results are presented in Table 1.

Regardless of their suitability for pharmaceuticals and cosmetics, all compounds were tested for their inhibition of melanin synthesis $\left(\mathrm{IC}_{50}\right)$. From the MTT assay, several 2-hydroxylsubstituted compounds exhibited moderate cytotoxicity. On the other hand, 3- or 4-hydroxy-substituted compounds showed low cytotoxicity $\left(\mathrm{IC}_{50}>100 \mu \mathrm{M}\right)$ as a cosmetic ingredient.

The effect of mono-hydroxycinnamic esters on melanin synthesis in B16 melanoma cells was investigated. For 4-hydroxycinnamic esters, electron-donating substituted benzylic cinnamic esters (1-4) showed potent inhibitory activity compared to those containing electron-withdrawing substituents $(\mathbf{5}, \mathbf{6})$. However, 3-hydroxycinnamic esters (9-15) displayed much weaker inhibitory activity and the opposite electronic effect, compared to the 4-hydroxycinnamic ester derivatives. Although several 2-hydroxycinnamic esters showed potent inhibitory activity, many of these exhibited severe cytotoxicity at low concentrations. The 4- and 3-hydroxycinnamic esters $(\mathbf{8}, \mathbf{1 6})$, which were extended by one carbon at the benzylic position, displayed excellent inhibitory activity compared to the corresponding benzylic cinnamic esters. These biological results revealed that the inhibitory activity of substituted benzyl esters depended on the position of the hydroxyl group on the phenyl ring of the cinnamic acid. In addition, mono-hydroxycinnamic

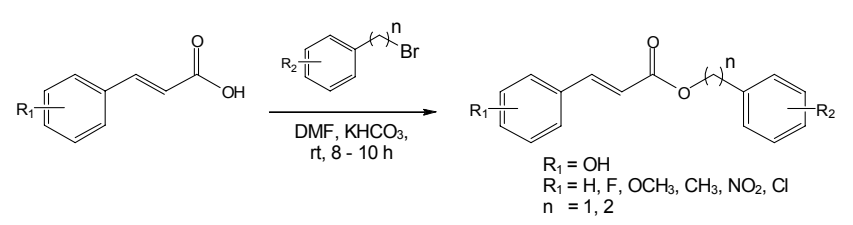

Figure 1. General synthetic pathway for the production of hydroxycinnamic esters from hydroxycinnamic acid. 
Table 1. Inhibition of melanin synthesis by hydroxycinnamic ester derivatives

\begin{tabular}{|c|c|c|c|c|c|c|c|}
\hline & $\mathrm{R}_{1}$ & $\mathrm{R}_{2}$ & $\mathrm{n}$ & $\begin{array}{c}\% \\
\text { Inhibition } \\
\text { at } 10 \mu \mathrm{M}\end{array}$ & $\begin{array}{c}\% \\
\text { Inhibition } \\
\text { at } 100 \mu \mathrm{M}\end{array}$ & $\begin{array}{c}\mathrm{IC}_{50} \\
(\mu \mathrm{M})\end{array}$ & $\begin{array}{c}\text { Cyto- } \\
\text { toxicity } \\
\mathrm{IC}_{50}(\mu \mathrm{M})\end{array}$ \\
\hline 1 & $4-\mathrm{OH}$ & 4-H & 1 & 35 & & 35 & $>100$ \\
\hline 2 & $4-\mathrm{OH}$ & $4-\mathrm{OCH}_{3}$ & 1 & 38 & & 14 & $>100$ \\
\hline 3 & $4-\mathrm{OH}$ & $2-\mathrm{CH}_{3}$ & 1 & 43 & & 13 & $>100$ \\
\hline 4 & $4-\mathrm{OH}$ & $4-\mathrm{CH}_{3}$ & 1 & 24 & & 27 & $>100$ \\
\hline 5 & 4-OH & $4-\mathrm{F}$ & 1 & 20 & & 98 & $>100$ \\
\hline 6 & $4-\mathrm{OH}$ & $4-\mathrm{Cl}$ & 1 & 3 & & $-{ }^{a}$ & 82 \\
\hline 7 & $4-\mathrm{OH}$ & $4-\mathrm{NO}_{2}$ & 1 & 19 & & 37 & $>100$ \\
\hline 8 & $4-\mathrm{OH}$ & 4-H & 2 & 61 & & 3 & $>100$ \\
\hline 9 & $3-\mathrm{OH}$ & 4-H & 1 & 14 & 18 & $-^{a}$ & $>100$ \\
\hline 10 & $3-\mathrm{OH}$ & $4-\mathrm{OCH}_{3}$ & 1 & 16 & 32 & $-{ }^{a}$ & $>100$ \\
\hline 11 & $3-\mathrm{OH}$ & $2-\mathrm{CH}_{3}$ & 1 & 8 & 16 & $-{ }^{a}$ & $>100$ \\
\hline 12 & $3-\mathrm{OH}$ & $4-\mathrm{CH}_{3}$ & 1 & 37 & 45 & $-{ }^{a}$ & $>100$ \\
\hline 13 & $3-\mathrm{OH}$ & $4-\mathrm{F}$ & 1 & 5 & 36 & $-{ }^{a}$ & $>100$ \\
\hline 14 & $3-\mathrm{OH}$ & $4-\mathrm{Cl}$ & 1 & 37 & & 20 & $>100$ \\
\hline 15 & $3-\mathrm{OH}$ & $4-\mathrm{NO}_{2}$ & 1 & 33 & & 85 & $>100$ \\
\hline 16 & $3-\mathrm{OH}$ & 4-H & 2 & 56 & & 8 & $>100$ \\
\hline 17 & $2-\mathrm{OH}$ & 4-H & 1 & 1 & 5 & $-{ }^{a}$ & $>100$ \\
\hline 18 & $2-\mathrm{OH}$ & $4-\mathrm{OCH}_{3}$ & 1 & 20 & & $-{ }^{a}$ & 83 \\
\hline 19 & $2-\mathrm{OH}$ & $2-\mathrm{CH}_{3}$ & 1 & 33 & & 16 & 31 \\
\hline 20 & $2-\mathrm{OH}$ & 4- $\mathrm{CH}_{3}$ & 1 & 16 & & $-{ }^{a}$ & 32 \\
\hline 21 & $2-\mathrm{OH}$ & $4-\mathrm{F}$ & 1 & 8 & & $-{ }^{a}$ & 63 \\
\hline 22 & $2-\mathrm{OH}$ & $4-\mathrm{Cl}$ & 1 & 15 & & 88 & $>100$ \\
\hline 23 & $2-\mathrm{OH}$ & $4-\mathrm{NO}_{2}$ & 1 & 43 & & 14 & $>100$ \\
\hline 24 & $2-\mathrm{OH}$ & $4-\mathrm{H}$ & 2 & 3 & & $-{ }^{a}$ & 34 \\
\hline \multicolumn{5}{|c|}{ 4-Hydroxycinnamic acid } & 38 & 127 & \\
\hline \multicolumn{5}{|c|}{ 3-Hydroxycinnamic acid } & 12 & $>400$ & \\
\hline \multicolumn{5}{|c|}{ 2-Hydroxycinnamic acid } & 22 & 359 & \\
\hline \multicolumn{5}{|c|}{ Arbutin } & 37 & 147 & \\
\hline \multicolumn{5}{|c|}{ Kojic acid } & 15 & 369 & \\
\hline
\end{tabular}

${ }^{a} \mathrm{IC}_{50}$ value not shown within the range of non-cytotoxic concentration.

esters, with specific substituents on the benzyl ester components, exhibited greater inhibition of melanogenesis than their corresponding hydroxycinnamic acids. The results demonstrated that benzylic 4-hydroxycinnamic esters have strong inhibitory activities on melanin synthesis and low cytotoxicity, compared to benzylic 2- or 3-hydroxycinnamic esters.

Since tyrosinase is known to be the enzyme responsible for the oxidation of tyrosine, the first and rate-limiting step in melanin synthesis, ${ }^{20}$ the effective compounds (1-5, 7-8, 14-16, and 22-23) reported in this study were evaluated for their tyrosinase inhibitory activity by using the mushroom tyrosinase assay. The substituted compounds $5\left(\mathrm{IC}_{50}=71 \mu \mathrm{M}\right)$ showed stronger inhibitory activity than kojic acid $\left(\mathrm{IC}_{50}=212 \mu \mathrm{M}\right)$. The other compounds not showed $\mathrm{IC}_{50}$ values within the range of non-cytotoxic concentration $(100 \mu \mathrm{M})$. The results revealed the inhibition activity on melanin synthesis of our substituted compounds may not be related to the direct inhibition of tyrosinase activity.

\section{Conclusions}

The esterification of mono-hydroxycinnamic acid by substitution with specific benzyl groups could increase the inhibitory activity on melanin synthesis. Specifically, benzylic 4-hydroxycinnamic esters displayed excellent inhibition of melanin synthesis, compared to kojic acid $\left(\mathrm{IC}_{50}=367 \mu \mathrm{M}\right)$ or arbutin $\left(\mathrm{IC}_{50}=147 \mu \mathrm{M}\right)$, which are well known depigmenting agents. Although these findings are too limited and premature for direct cosmetic applications, they provide a general direction for future research on hydroxycinnamic esters as effective depigmenting agents. Future researches on their more detail inhibitory activities in melanin synthesis and in vivo test are under way.

\section{Experimental Section}

Melting points were determined using a Thermo Scientific Electro thermal 9100 melting point apparatus. ${ }^{1} \mathrm{H}$ and ${ }^{13} \mathrm{C}$ NMR spectra were recorded on a JEOL JNM-AL400 spectrometer (400 MHz for ${ }^{1} \mathrm{H}$ and $100 \mathrm{MHz}$ for ${ }^{13} \mathrm{C}$ ). Mass spectra were obtained using a Waters Quattro premier XE mass spectrometer. UV spectra were collected using a Molecular Devices Spectra Max microplate reader.

Materials. Kojic acid (5-hydroxy-2-(hydroxymethyl)- $4 H$ pyran-4-one), $o$-hydroxycinnamic acid, $m$-hydroxycinnamic acid, $p$-hydroxycinnamic acid, MTT (3-(4,5-dimethylthiazol2-yl)-2,5-diphenyltetrazolium bromide) was purchased from Aldrich Chemical Co. All other chemicals and solvents were used with the highest grade commercially available.

General procedure for the synthesis of compound $1 .^{21} p$ Hydroxy cinnamic acid ( $328 \mathrm{mg}, 2.0 \mathrm{mmol}$ ) was dissolved in $10.0 \mathrm{~mL}$ DMF, and $\mathrm{KHCO}_{3}(240 \mathrm{mg}, 2.4 \mathrm{mmol}$ ) was added slowly. The resultant mixture was stirred for several minutes at room temperature. Then, benzyl bromide $(513 \mathrm{mg}, 3.0 \mathrm{mmol})$ was added and stirred for $8-10 \mathrm{~h}$ at room temperature. Upon completion, the reaction mixture was added to water and extracted with ethyl acetate. The organic layer was washed with brine and dried over anhydrous magnesium sulfate. The solution was filtered and concentrated. The residue was purified by column chromatography using ethyl acetate-hexane (3:1) as an eluent. The following compounds were prepared by this general procedure.

Benzyl 3-(4-hydroxyphenyl)acrylate (1). Yield 65\%; white solid; mp $90-92{ }^{\circ} \mathrm{C} ;{ }^{1} \mathrm{H}$ NMR $\left(400 \mathrm{MHz}, \mathrm{CDCl}_{3}\right) \delta 7.66(\mathrm{~d}$, $1 \mathrm{H}, J=16.0 \mathrm{~Hz}), 7.40-7.30(\mathrm{~m}, 7 \mathrm{H}), 6.84(\mathrm{~d}, 2 \mathrm{H}, J=8.0 \mathrm{~Hz})$, $6.32(\mathrm{~d}, 1 \mathrm{H}, J=16.0 \mathrm{~Hz}), 5.24(\mathrm{~s}, 2 \mathrm{H}) ;{ }^{13} \mathrm{C}$ NMR $(100 \mathrm{MHz}$, $\left.\mathrm{CDCl}_{3}\right) \delta 168.1,158.5,145.7,135.7,130.1,128.6,128.2,128.1$, 126.4, 115.9, 114.4, 66.5; MS $m / z 253[\mathrm{M}-\mathrm{H}]^{-}$.

4-Methoxybenzyl 3-(4-hydroxyphenyl)acrylate (2). Yield $53 \%$; white solid; mp $90-92{ }^{\circ} \mathrm{C} ;{ }^{1} \mathrm{H}$ NMR ( $400 \mathrm{MHz}$, acetone- $d_{6}$ ) $\delta 7.64(\mathrm{~d}, 1 \mathrm{H}, J=16.0 \mathrm{~Hz}), 7.54$ (d, 2H, $J=8.8 \mathrm{~Hz}), 7.37$ (d, 2H, $J=8.4 \mathrm{~Hz}), 6.93(\mathrm{~d}, 2 \mathrm{H}, J=8.4 \mathrm{~Hz}), 6.89(\mathrm{~d}, 2 \mathrm{H}, J=8.8 \mathrm{~Hz})$, 6.37 (d, $1 \mathrm{H}, J=16.0 \mathrm{~Hz}$ ), 5.12 (s, $2 \mathrm{H}$ ), 3.79 (s. $3 \mathrm{H}),{ }^{13} \mathrm{C} \mathrm{NMR}$ $\left(100 \mathrm{MHz}\right.$, acetone- $\left.d_{6}\right) \delta 167.3,160.6,145.5,130.9,130.8$, 129.6, 126.9, 116.6, 115.5, 114.6, 66.1, 55.5; MS m/z 283 $[\mathrm{M}-\mathrm{H}]^{-}$.

2-Methylbenzyl 3-(4-hydroxyphenyl)acrylate (3). Yield $50 \%$; white solid; mp $86-88^{\circ} \mathrm{C} ;{ }^{1} \mathrm{H}$ NMR $\left(400 \mathrm{MHz}\right.$, acetone- $\left.d_{6}\right)$ $\delta 7.66(\mathrm{~d}, 1 \mathrm{H}, J=16.0 \mathrm{~Hz}), 7.55(\mathrm{~d}, 2 \mathrm{H}, J=8.8 \mathrm{~Hz}), 7.39(\mathrm{~d}$, $1 \mathrm{H}, J=7.2 \mathrm{~Hz}), 7.25-7.20(\mathrm{~m}, 3 \mathrm{H}), 6.90(\mathrm{~d}, 2 \mathrm{H}, J=8.8 \mathrm{~Hz}), 6.41$ $(\mathrm{d}, 1 \mathrm{H}, J=16.0 \mathrm{~Hz}), 5.23(\mathrm{~s}, 2 \mathrm{H}), 2.37(\mathrm{~s}, 3 \mathrm{H}),{ }^{13} \mathrm{C}$ NMR $(100$ 
MHz, acetone- $\left.d_{6}\right) \delta 167.2,160.6,145.6,137.6,135.4,131.0$, $130.9,129.9,129.1,126.9,126.7,116.6,115.2,64.7,18.9 ; \mathrm{MS}$ $\mathrm{m} / \mathrm{z} 267[\mathrm{M}-\mathrm{H}]^{-}$.

4-Methylbenzyl 3-(4-hydroxyphenyl)acrylate(4). Yield 63\%; white solid; mp 106 - $108{ }^{\circ} \mathrm{C}$; ${ }^{1} \mathrm{H}$ NMR $\left(400 \mathrm{MHz}, \mathrm{CDCl}_{3}\right) \delta 7.67$ $(\mathrm{d}, 1 \mathrm{H}, J=16.0 \mathrm{~Hz}), 7.37(\mathrm{~d}, 2 \mathrm{H}, J=8.8 \mathrm{~Hz}), 7.30(\mathrm{~d}, 2 \mathrm{H}, J=$ $8.4 \mathrm{~Hz}), 7.18(\mathrm{~d}, 2 \mathrm{H}, J=8.4 \mathrm{~Hz}), 6.84(\mathrm{~d}, 2 \mathrm{H}, J=8.8 \mathrm{~Hz}), 6.32$ $(\mathrm{d}, 1 \mathrm{H}, J=16.0 \mathrm{~Hz}), 5.21(\mathrm{~s}, 2 \mathrm{H}), 2.35(\mathrm{~s}, 3 \mathrm{H}) ;{ }^{13} \mathrm{C}$ NMR $(100$ $\left.\mathrm{MHz}, \mathrm{CDCl}_{3}\right) \delta 167.7,158.0,145.2,138.1,132.9,130.0,129.3$, 128.4, 126.9, 115.9, 115.0, 66.4, 21.2; MS m/z 267 [M-H] .

4-Fluorobenzyl 3-(4-hydroxyphenyl)acrylate (5). Yield 65\%; white solid; mp $99-101{ }^{\circ} \mathrm{C} ;{ }^{1} \mathrm{H}$ NMR $\left(400 \mathrm{MHz}, \mathrm{CDCl}_{3}\right) \delta 7.67$ $(\mathrm{d}, 1 \mathrm{H}, J=16.0 \mathrm{~Hz}), 7.44-7.38(\mathrm{~m}, 4 \mathrm{H}), 7.09-7.05(\mathrm{~m}, 2 \mathrm{H}), 6.85$ $(\mathrm{d}, 2 \mathrm{H}, J=8.8 \mathrm{~Hz}), 6.34(\mathrm{~d}, 1 \mathrm{H}, J=16.0 \mathrm{~Hz}), 5.21(\mathrm{~s}, 2 \mathrm{H}) ;{ }^{13} \mathrm{C}$ NMR $\left(100 \mathrm{MHz} \mathrm{CDCl}_{3}\right) \delta 168.1,163.8,161.4,158.5,145.8$, $131.6,130.2,130.1,126.4,115.9,115.5,115.3,114.2,65.8 ; \mathrm{MS}$ $\mathrm{m} / \mathrm{z} 271[\mathrm{M}-\mathrm{H}]^{-}$.

4-Chlorobenzyl 3-(4-hydroxyphenyl)acrylate (6). Yield 43\%; white solid; mp $114-116{ }^{\circ} \mathrm{C} ;{ }^{1} \mathrm{H}$ NMR (400 MHz, acetone- $\left.d_{6}\right)$ $\delta 7.67(\mathrm{~d}, 1 \mathrm{H}, J=16.0 \mathrm{~Hz}), 7.56(\mathrm{~d}, 2 \mathrm{H}, J=8.8 \mathrm{~Hz}), 7.47-7.37$ $(\mathrm{m}, 4 \mathrm{H}), 6.91(\mathrm{~d}, 1 \mathrm{H}, J=8.8 \mathrm{~Hz}), 6.42(\mathrm{~d}, 1 \mathrm{H}, J=16.0 \mathrm{~Hz}), 5.22$ (s, 2H); ${ }^{13} \mathrm{C}$ NMR (100 MHz, acetone- $\left.d_{6}\right) \delta 167.1,160.6,145.9$, $136.6,134.1,131.0,130.6,129.3,126.8,116.6,115.0,65.4 ; \mathrm{MS}$ $\mathrm{m} / \mathrm{z} 287[\mathrm{M}-\mathrm{H}]^{-}$.

4-Nitrobenzyl 3-(4-hydroxyphenyl)acrylate (7). Yield 56\%; white solid; mp $151-153{ }^{\circ} \mathrm{C} ;{ }^{1} \mathrm{H}$ NMR (400 MHz, acetone- $d_{6}$ ) $\delta 8.18(\mathrm{~d}, 2 \mathrm{H}, J=8.4 \mathrm{~Hz}), 7.67-7.61(\mathrm{~m}, 3 \mathrm{H}), 7.50(\mathrm{~d}, 2 \mathrm{H}, J=$ $8.8 \mathrm{~Hz}), 6.86(\mathrm{~d}, 2 \mathrm{H}, J=8.4 \mathrm{~Hz}), 6.40(\mathrm{~d}, 1 \mathrm{H}, J=16.0 \mathrm{~Hz})$, $5.32(\mathrm{~s}, 2 \mathrm{H}) ;{ }^{13} \mathrm{C}$ NMR $\left(100 \mathrm{MHz}\right.$, acetone- $\left.d_{6}\right) \delta 167.1,160.7$, 148.4, 146.2, 145.2, 131.1, 129.1, 126.7, 124.2, 116.7, 114.6, 65.0; MS $m / z 298$ [M-H] .

Phenethyl 3-(4-hydroxyphenyl)acrylate (8). Yield 27\%; white solid; mp 90 - $92{ }^{\circ} \mathrm{C} ;{ }^{1} \mathrm{H}$ NMR (400 MHz, CD $\left.{ }_{3} \mathrm{OD}\right) \delta 7.57$ $(\mathrm{d}, 1 \mathrm{H}, J=16.0 \mathrm{~Hz}), 7.38(\mathrm{~d}, 2 \mathrm{H}, J=8.8 \mathrm{~Hz}), 7.29-7.17(\mathrm{~m}, 6 \mathrm{H})$, $6.85(\mathrm{~d}, 1 \mathrm{H}, J=8.8 \mathrm{~Hz}), 6.25(\mathrm{~d}, 1 \mathrm{H}, J=16.0 \mathrm{~Hz}), 4.33(\mathrm{t}, 2 \mathrm{H}$, $J=7.0 \mathrm{~Hz}), 2.94(\mathrm{t}, 2 \mathrm{H}, J=7.0 \mathrm{~Hz}) ;{ }^{13} \mathrm{C}$ NMR $(100 \mathrm{MHz}$, $\left.\mathrm{CD}_{3} \mathrm{OD}\right) \delta 169.2,161.2,146.6,139.3,131.3,130.0,129.5,127.6$, 127.1, 116.9, 115.2, 66.1, 36.1; MS m/z 267 [M-H] .

Benzyl 3-(3-hydroxyphenyl)acrylate (9). Yield 59\%; white solid; mp 93 - $95{ }^{\circ} \mathrm{C} ;{ }^{1} \mathrm{H}$ NMR (400 MHz, $\left.\mathrm{CDCl}_{3}\right) \delta 7.54$ (d, $1 \mathrm{H}, J=16.0 \mathrm{~Hz}), 7.29-7.20(\mathrm{~m}, 5 \mathrm{H}), 7.08(\mathrm{t}, 1 \mathrm{H}, J=8.2 \mathrm{~Hz})$, 6.91-6.89 (m, 2H), $6.78(\mathrm{~d}, 1 \mathrm{H}, J=8.0 \mathrm{~Hz}), 6.30(\mathrm{~d}, 1 \mathrm{H}, J=$ $16.0 \mathrm{~Hz}), 5.22(\mathrm{~s}, 2 \mathrm{H}) ;{ }^{13} \mathrm{C} \mathrm{NMR}\left(100 \mathrm{MHz}, \mathrm{CDCl}_{3}\right) \delta 167.5$, $156.3,145.6,135.6,135.5,130.0,128.5,128.3,128.2,120.6$, 117.8, 117.6, 114.6, 66.7; MS m/z 253 [M-H]

4-Methoxybenzyl 3-(3-hydroxyphenyl)acrylate (10). Yield $60 \%$; white solid; mp $108-110{ }^{\circ} \mathrm{C} ;{ }^{1} \mathrm{H}$ NMR (400 MHz, acetone- $\left.d_{6}\right) \delta 7.64(\mathrm{~d}, 1 \mathrm{H}, J=16.0 \mathrm{~Hz}), 7.38(\mathrm{~d}, 2 \mathrm{H}, J=8.0 \mathrm{~Hz})$, $7.25(\mathrm{t}, 1 \mathrm{H}, J=7.8 \mathrm{~Hz}), 7.15-7.13(\mathrm{~m}, 2 \mathrm{H}), 6.94-6.91(\mathrm{~m}, 3 \mathrm{H})$, $6.50(\mathrm{~d}, 1 \mathrm{H}, J=16.0 \mathrm{~Hz}), 5.16(\mathrm{~s}, 2 \mathrm{H}), 3.79(\mathrm{~s}, 3 \mathrm{H}) ;{ }^{13} \mathrm{C} \mathrm{NMR}$ $\left(100 \mathrm{MHz}\right.$, acetone- $\left.d_{6}\right) \delta 167.0,160.6,158.6,145.5,136.7$, $130.9,130.8,129.3,120.5,118.8,118.4,115.3,114.6,66.4$, 55.5; MS $m / z 283[\mathrm{M}-\mathrm{H}]^{-}$.

2-Methylbenzyl 3-(3-hydroxyphenyl)acrylate (11). Yield 68\%; white solid; mp $94-96{ }^{\circ} \mathrm{C} ;{ }^{1} \mathrm{H}$ NMR (400 MHz, $\mathrm{CDCl}_{3}$ ) $\delta 7.69(\mathrm{~d}, 1 \mathrm{H}, J=16.0 \mathrm{~Hz}), 7.39(\mathrm{~d}, 1 \mathrm{H}, J=7.0 \mathrm{~Hz}), 7.31-7.22$ $(\mathrm{m}, 4 \mathrm{H}), 7.07-7.06(\mathrm{~m}, 2 \mathrm{H}), 6.94(\mathrm{~d}, 1 \mathrm{H}, J=8.0 \mathrm{~Hz}), 6.46(\mathrm{~d}$, $1 \mathrm{H}, J=16.0 \mathrm{~Hz}), 5.32(\mathrm{~s}, 2 \mathrm{H}), 2.40(\mathrm{~s}, 3 \mathrm{H}) ;{ }^{13} \mathrm{C}$ NMR $(100$
$\left.\mathrm{MHz}, \mathrm{CDCl}_{3}\right) \delta 167.9,156.6,145.9,137.2,135.7,133.7,130.6$, 130.2, 129.4, 128.8, 126.2, 120.9, 118.1, 117.7, 114.9, 65.4, 19.0; MS $m / z 267$ [M-H] .

4-Methylbenzyl 3-(3-hydroxyphenyl)acrylate (12). Yield $73 \%$; white solid; mp $101-103{ }^{\circ} \mathrm{C}$; ${ }^{1} \mathrm{H}$ NMR (400 MHz, acetone- $\left.d_{6}\right) \delta 7.64(\mathrm{~d}, 1 \mathrm{H}, J=16.0 \mathrm{~Hz}), 7.34(\mathrm{~d}, 2 \mathrm{H}, J=8.0 \mathrm{~Hz})$, $7.26(\mathrm{t}, 1 \mathrm{H}, J=8.0 \mathrm{~Hz}), 7.21(\mathrm{~d}, 2 \mathrm{H}, J=8.0 \mathrm{~Hz}), 7.18-7.12(\mathrm{~m}$, $2 \mathrm{H}), 6.92(\mathrm{~d}, 1 \mathrm{H}, J=8.0 \mathrm{~Hz}), 6.52(\mathrm{~d}, 1 \mathrm{H}, J=16.0 \mathrm{~Hz}), 5.19$ (s, 2H), 2.33 (s. 3H); ${ }^{13} \mathrm{C}$ NMR (100 MHz, $\left.\mathrm{CDCl}_{3}\right) \delta 167.7$, 156.3, 145.5, 138.2, 135.5, 132.5, 130.0, 129.2, 128.4, 120.6, 117.8, 117.6, 114.6, 66.7, 21.1; MS m/z 267 [M-H].

4-Fluorobenzyl 3-(3-hydroxyphenyl)acrylate (13). Yield 68\%; white solid; mp $105-107{ }^{\circ} \mathrm{C} ;{ }^{1} \mathrm{H} \mathrm{NMR}\left(400 \mathrm{MHz}, \mathrm{CDCl}_{3}\right)$ $\delta 7.66(\mathrm{~d}, 1 \mathrm{H}, J=16.0 \mathrm{~Hz}), 7.42-7.38(\mathrm{~m}, 2 \mathrm{H}), 7.22(\mathrm{t}, 1 \mathrm{H}, J=$ $7.8 \mathrm{~Hz}), 7.10-7.00(\mathrm{~m}, 4 \mathrm{H}), 6.88(\mathrm{~d}, 1 \mathrm{H}, J=8.0 \mathrm{~Hz}), 6.44$ (d, $1 \mathrm{H}, J=16.0 \mathrm{~Hz}), 5.21(\mathrm{~s}, 2 \mathrm{H}) ;{ }^{13} \mathrm{C} \mathrm{NMR}\left(100 \mathrm{MHz}, \mathrm{CDCl}_{3}\right) \delta$ $167.4,163.9,161.4,159.5,145.6,135.5,131.5,130.2$, 130.1, 120.7, 117.8, 117.6, 115.6, 115.4, 114.6, 65.9; MS m/z 271 $[\mathrm{M}-\mathrm{H}]^{-}$.

4-Chlorobenzyl 3-(3-hydroxyphenyl)acrylate (14). Yield 48\%; white solid; mp $158-160{ }^{\circ} \mathrm{C}$; ${ }^{1} \mathrm{H}$ NMR (400 MHz, DMSO$\left.d_{6}\right) \delta 7.60(\mathrm{~d}, 1 \mathrm{H}, J=16.0 \mathrm{~Hz}), 7.45-7.38(\mathrm{~m}, 4 \mathrm{H}), 7.20(\mathrm{t}, 1 \mathrm{H}$, $J=7.8 \mathrm{~Hz}), 7.10-7.06(\mathrm{~m}, 2 \mathrm{H}), 6.85(\mathrm{~d}, 1 \mathrm{H}, J=8.0 \mathrm{~Hz}), 6.54(\mathrm{~d}$, $1 \mathrm{H}, J=16.0 \mathrm{~Hz}) 5.18(\mathrm{~s}, 2 \mathrm{H}) ;{ }^{13} \mathrm{C}$ NMR $\left(100 \mathrm{MHz}, \mathrm{DMSO}-d_{6}\right)$ $\delta 166.2,157.9,145.4,135.4,135.3,132.9,130.1,130.0,128.6$, 119.6, 118.0, 117.5, 114.8, 65.0; MS m/z 287 [M-H] .

4-Nitrobenzyl 3-(3-hydroxyphenyl)acrylate (15). Yield 57\%; white solid; mp $135-137{ }^{\circ} \mathrm{C} ;{ }^{1} \mathrm{H}$ NMR (400 MHz, acetone- $d_{6}$ ) $\delta 8.19(\mathrm{~d}, 2 \mathrm{H}, J=8.0 \mathrm{~Hz}), 7.67-7.63(\mathrm{~m}, 3 \mathrm{H}), 7.21(\mathrm{t}, 1 \mathrm{H}, J=7.8$ $\mathrm{Hz}), 7.11-7.09$ (m, 2H), $6.88(\mathrm{~d}, 1 \mathrm{H}, J=8.0 \mathrm{~Hz}), 6.52(\mathrm{~d}, 1 \mathrm{H}$, $J=16.0 \mathrm{~Hz}), 5.33(\mathrm{~s}, 2 \mathrm{H}) ;{ }^{13} \mathrm{C}$ NMR $\left(100 \mathrm{MHz}\right.$, acetone- $\left.d_{6}\right) \delta$ $166.7,158.7,148.4,146.3,145.0,136.5,130.8,129.3,124.3$, 120.6, 118.6, 118.1, 115.4, 65.2; MS $m / z 298$ [M-H]

Phenethyl 3-(3-hydroxyphenyl)acrylate (16). Yield 27\%; white solid; mp $78-79{ }^{\circ} \mathrm{C} ;{ }^{1} \mathrm{H}$ NMR (400 MHz, CD $\left.3 \mathrm{OD}\right) \delta$ $7.56(\mathrm{~d}, 1 \mathrm{H}, J=16.0 \mathrm{~Hz}), 7.29-7.17(\mathrm{~m}, 6 \mathrm{H}), 7.00-6.99(\mathrm{~m}, 2 \mathrm{H})$, $6.85(\mathrm{~d}, 1 \mathrm{H}, J=8.0 \mathrm{~Hz}), 6.39(\mathrm{~d}, 1 \mathrm{H}, J=16.0 \mathrm{~Hz}), 4.35(\mathrm{t}, 2 \mathrm{H}$, $J=7.0 \mathrm{~Hz}), 2.96(\mathrm{t}, 2 \mathrm{H}, J=7.0 \mathrm{~Hz}) ;{ }^{13} \mathrm{C} \mathrm{NMR}(100 \mathrm{MHz}$, $\left.\mathrm{CD}_{3} \mathrm{OD}\right) \delta 168.6,159.0,146.5,139.3,136.9,130.0,129.5,127.6$, 120.9, 118.6, 115.4, 66.3, 36.1; MS $m / z 267$ [M-H] .

Benzyl 3-(2-hydroxyphenyl)acrylate (17). Yield 58\%; white solid; mp $86-88{ }^{\circ} \mathrm{C} ;{ }^{1} \mathrm{H}$ NMR $\left(400 \mathrm{MHz}, \mathrm{CDCl}_{3}\right) \delta 8.12(\mathrm{~d}$, $1 \mathrm{H}, J=16.0 \mathrm{~Hz}), 7.40-7.27(\mathrm{~m}, 6 \mathrm{H}), 7.16(\mathrm{t}, 1 \mathrm{H}, J=8.4 \mathrm{~Hz})$, 6.85-6.79 (m, 2H), $6.71(\mathrm{~d}, 1 \mathrm{H}, J=16.0 \mathrm{~Hz}), 5.25(\mathrm{~s}, 2 \mathrm{H}) ;{ }^{13} \mathrm{C}$ NMR $\left(100 \mathrm{MHz} \mathrm{CDCl}_{3}\right) \delta 168.7,155.9,141.9,135.8,131.6$, 129.3, 128.5, 128.2, 128.1, 121.4, 120.3, 117.4, 116.4, 66.7; MS $m / z 253[\mathrm{M}-\mathrm{H}]^{-}$.

4-Methoxybenzyl 3-(2-hydroxyphenyl)acrylate (18). Yield 64\%; white solid; mp $128-130{ }^{\circ} \mathrm{C} ;{ }^{1} \mathrm{H}$ NMR (400 MHz, acetone- $\left.d_{6}\right) \delta 8.04(\mathrm{~d}, 1 \mathrm{H}, J=16.0 \mathrm{~Hz}), 7.58(\mathrm{~d}, 1 \mathrm{H}, J=8.0 \mathrm{~Hz})$, $7.38(\mathrm{~d}, 2 \mathrm{H}, J=8.0 \mathrm{~Hz}), 7.24(\mathrm{t}, 1 \mathrm{H}, J=7.6 \mathrm{~Hz}), 6.99-6.92(\mathrm{~m}$, $3 \mathrm{H}), 6.88(\mathrm{t}, 1 \mathrm{H}, J=7.6 \mathrm{~Hz}), 6.66(\mathrm{~d}, 1 \mathrm{H}, J=16.0 \mathrm{~Hz}), 5.16(\mathrm{~s}$, $2 \mathrm{H}), 3.78(\mathrm{~s}, 3 \mathrm{H}) ;{ }^{13} \mathrm{C}-\mathrm{NMR}\left(100 \mathrm{MHz}\right.$, acetone- $\left.d_{6}\right) \delta 167.5$, $160.5,157.4,141.1,132.2,130.8,129.7,129.5,122.2$, 120.7, 118.5, 117.0, 114.6, 66.2, 55.5; MS m/z 283 [M-H] .

2-Methylbenzyl 3-(2-hydroxyphenyl)acrylate (19). Yield 61\%; white solid; mp $110-112{ }^{\circ} \mathrm{C} ;{ }^{1} \mathrm{H} \mathrm{NMR}\left(400 \mathrm{MHz}, \mathrm{CDCl}_{3}\right)$ $\delta 8.12(\mathrm{~d}, 1 \mathrm{H}, J=16.0 \mathrm{~Hz}), 7.42-7.37(\mathrm{~m}, 2 \mathrm{H}), 7.27-7.16(\mathrm{~m}$, 
$4 \mathrm{H}), 6.86(\mathrm{t}, 1 \mathrm{H}, J=7.2 \mathrm{~Hz}), 6.77(\mathrm{~d}, 1 \mathrm{H}, J=8.0 \mathrm{~Hz}), 6.73(\mathrm{~d}$, $1 \mathrm{H}, J=16.0 \mathrm{~Hz}), 5.28(\mathrm{~s}, 2 \mathrm{H}), 2.37(\mathrm{~s}, 3 \mathrm{H}) ;{ }^{13} \mathrm{C} \mathrm{NMR}(100 \mathrm{MHz}$, $\left.\mathrm{CDCl}_{3}\right) \delta 168.9,155.9,141.8,137.0,133.7,131.6,130.4,129.3$, 128.6, 126.0, 121.4, 120.4, 117.4, 116.4, 65.1, 18.9; MS m/z $267[\mathrm{M}-\mathrm{H}]^{-}$.

Cell culture. B16F1 murine melanoma cells were purchased from the American Type Culture Collection. The cells were grown in DMEM (Gibco BRL, Grand Island, NY, USA) supplemented with 10\% fetal bovine serum (Gibco BRL) and 1\% penicillin-streptomycin (Gibco BRL). The cells were maintained in a humidified incubator with $5 \% \mathrm{CO}_{2}$ at $37{ }^{\circ} \mathrm{C}$. All of the tested compounds were prepared in DMSO.

MTT assay. The MTT assay has been described by Mosmann. ${ }^{22,23}$ Briefly, a cell suspension was seeded in a microplate, and the cells were allowed to adhere completely to the plate overnight. Each test compound was added to the plate and then incubated at $37^{\circ} \mathrm{C}$ in a $\mathrm{CO}_{2}$ incubator. After $24 \mathrm{~h}, 10 \mu \mathrm{L}$ of MTT solution $(5 \mathrm{mg} / \mathrm{mL}$ in PBS) was added to each well, and the incubation was continued for $4 \mathrm{~h}$. The supernatant was then removed, and formazan was solubilized by adding $100 \mu \mathrm{L}$ of DMSO to each well with gentle shaking. The absorbance was then measured using a microplate reader at $570 \mathrm{~nm}$.

Melanin content assay. The melanin content was measured using the method reported by Shimizu et al., ${ }^{24}$ with a slight modification. Briefly, B16F1 murine melanoma cells were rinsed in PBS and removed using $0.05 \%$ trypsin/EDTA. The cells were incubated at density $1 \times 10^{5}$ cells in six-well plates overnight. The cells were treated with the test compound at various concentrations in phenol red-free DMEM for $3 \mathrm{~d}$. After removing the medium and washing the cells with PBS, the cells were dissolved in $1 \mathrm{~N} \mathrm{NaOH}$ containing 10\% DMSO. The relative melanin content was determined by optical density (OD) at 405 $\mathrm{nm}$ using a microplate reader.

Mushroom tyrosinase assay. The tyrosinase activity was determined using the method described by Masamoto et al. with a slight modification. ${ }^{25}$ The test compound solution was prepared by mixing the compound with DMSO and a $0.1 \mathrm{M}$ phosphate buffer ( $\mathrm{pH}$ 7.0). Then a test tube was filled with 80 $\mu \mathrm{L}$ of the $0.1 \mathrm{M}$ phosphate buffer ( $\mathrm{pH} 7.0), 40 \mu \mathrm{L}$ of the test compound solution, and $40 \mu \mathrm{L}$ of mushroom tyrosinase $(1,500$ $2,000) \mathrm{U} / \mu \mathrm{L}$. Then $40 \mu \mathrm{L}$ of $2.5 \mathrm{mM} \mathrm{L}$-dopa was added. The same control solution without the test compound was also prepared. The UV absorbance was measured using a microplate reader at $475 \mathrm{~nm}$, and the percent inhibitions (\%) were calculated using the following formula:

$$
\text { Percent inhibitions }(\%)=[(A c-A s) / A c] \times 100
$$

where $A c$ and $A s$ are the absorbance for the control and for the samples, respectively. Each sample was assayed at different concentrations.

Acknowledgments. The authors thank for Chungnam National University and Natural Solution Corporation financial support.

\section{References}

1. Choi, S. Y.; Hwang, J. S.; Kim, S. H.; Kim, S. Y. Biochem. Biophys. Res. Commun. 2002, 349, 39.

2. Baek, H. S.; Rho, H. S.; Yoo, J. W.; Ahn, S. M.; Lee, J. Y.; Lee, J. A.; Kim, M. K.; Kim, D. H.; Chang, I. S. Bull. Korean Chem. Soc. 2008, 29, 43.

3. Kim, Y. J.; No, J. K.; Lee, J. S.; Kim, M. S.; Chung, H. Y. Biosci. Biotechnol. Biochem. 2006, 70, 532.

4. Koo, J. H.; Kim, H. T.; Yoon, H. Y.; Kwon, K. B.; Choi, I. W.; Jung, S. H.; Kim, H. U.; Park, B. H.; Park, J. W. Exp. Mol. Med. 2008, 40, 313.

5. Sato, K.; Takahashi, H.; Iraha, R.; Toriyama, M. Biol. Pharm. Bull. 2008, 31, 33 .

6. Chun, H. J.; Jeong, S. I.; Woo, W. H.; Kim, I. K. Bull. Korean Chem. Soc. 2001, 22, 1159.

7. Jung, G. D.; Yang, J. Y.; Song, E. S.; Park, J. W. Exp. Mol. Med. 2001, 33, 131.

8. Masamoto, Y.; Murata, Y.; Baba, K.; Shimoishi, Y.; Toda, M.; Takahata, K. Biol. Pharm. Bull. 2004, 27, 422.

9. Rho, H. S.; Baek, H. S.; Ahn, S. M.; Yoo, J. W.; Kim, D. H.; Kim, H. G. Bull. Korean Chem. Soc. 2009, 30, 475.

10. Lin, J. W.; Chiang, H. M.; Lin, Y. C.; Wen, K. C. J. Food. Drug. Anal. 2008, 16, 1 .

11. Parvez, S.; Kang, M.; Chung, H. S.; Bae, H. Phytother. Res. 2007, 21,805 .

12. Hori, I.; Nihei, K.; Kubo, I. Phytother. Res. 2004, 18, 475.

13. Kim, D. H.; Hwang, J. S.; Beak, H. S.; Kim, K. J.; Lee, B. G.; Chang, I.; Kang, H. H.; Lee, O. S. Chem. Pharm. Bull. 2003, 51, 113.

14. Kim, K. D.; Song, M. H.; Yum, E. K.; Jeon, O. S.; Ju, Y. W.; Chang, M. S. Bull. Korean Chem. Soc. 2009, 30, 1619.

15. Lim, J. Y.; Ishiguro, K.; Kubo, I. Phytother. Res.1999, 13, 371.

16. Jung, H. W.; Choi, J. Y.; Lee, J. G.; Choi, E. H.; Oh, J. S.; Kim, D. C.; Kim, J. A.; Park, S. H.; Son, J. K.; Lee, S. H. Kor. J. Pharmacogn. 2007, 38, 382.

17. Shi, Y.; Chen, Q. X.; Wang, Q.; Song, K. K.; Qiu, L. Food Chem. 2005, 92,707 .

18. Battaini, G.; Monzani, E.; Casella, L.; Lonadi, E.; Tepper, A. W. J. W.; Canters, G. W.; Bubacco, L. J. Biol. Chem. 2002, 277, 44606.

19. Satsuya, K.; Ogawa, M.; Sugibayashi, K.; Yamada, K.; Yamamoto, K. Arch. Pharm. Res. 1999, 22, 335.

20. Kang, H. H.; Rho, H. S.; Hwang, J. S.; Oh, S. G. Chem. Pharm. Bull. 2003, 51, 1085.

21. Guo, W.; Li, J.; Fan, N.; Wu, W.; Zhou, P.; Xia, C. Synth Commun. $\mathbf{2 0 0 5}, 35,145$.

22. Mosmann, T. J. Immunol. Meth. 1983, 65, 55.

23. Russell, C. A.; Vindelov, L. L. J. Immunol. Meth. 1998, 217, 165.

24. Shimizu, K.; Fukunaga, S.; Yoshikawa, K.; Kondo, R. J. Wood. Sci. 2007, 53, 153.

25. Masamoto, Y.; Lisa, K.; Kubo, M. Planta. Med. 1980, 40, 361. 